\title{
Assessment of nutritional status and prognosis in surgical intensive care unit: The prognostic and inflammatory nutritional index (PINI)
}

\author{
Hedi Gharsallah $^{1}$, Zied Hajjej ${ }^{1}$, Imen Naas ${ }^{1}$, Zied Aouni $^{2}$, Nejla Stambouli ${ }^{2}$, Iheb Labbène ${ }^{1}$, \\ Mustapha Ferjani ${ }^{1}$ \\ ${ }^{1}$ Université de Tunis El Manar, Faculté de Médicine de Tunis, Hôpital militaire de Tunis, service d'anesthésie réanimation, 1008, Tunis, \\ Tunisie \\ ${ }^{2}$ Hôpital militaire de Tunis, service de biochimie, 1008, Tunis, Tunisie
}

\section{Email address:}

gharsallahhedi@gmail.com (H. Gharsallah)

\section{To cite this article:}

Hedi Gharsallah, Zied Hajjej, Imen Naas, Zied Aouni, Nejla Stambouli, Iheb Labbène, Mustapha Ferjani. Assessment of Nutritional Status and Prognosis in Surgical Intensive Care Unit: The Prognostic and Inflammatory Nutritional Index (PINI). International Journal of Nutrition and Food Sciences. Vol. 3, No. 5, 2014, pp. 477-483. doi: 10.11648/j.ijnfs.20140305.27

\begin{abstract}
Background Malnutrition is an independent factor of morbidity and mortality in patients at intensive care unit. The prognostic inflammatory and nutritional index (PINI) is frequently used as a marker of malnutrition but this scoring system was not studied in surgical intensive care units. The purposes of this study were to assist nutritional status with biomarkers and to search for a correlation between biological markers and prognosis, using the PINI. Methods A prospective, observational study was performed in the intensive care unit, started in July 2013. Twenty surgical patients, spent at least seven days at the intensive care unit were enrolled. An early nutritional care was given (first 24 hours). The patients were evaluated each week clinically and biologically (Albumin, Prealbumin, Orosomucoid, C-reactive protein) in order to establish the prognostic inflammatory and nutritional index (PINI = C-reactive protein $\mathrm{x}$ Orosomucoid / Albumin $\mathrm{x}$ Prealbumin). The correlation between variables was calculated, the Spearman rank-order correlation was used. Results The average age was 56+/-11 years, IGS II score was 48+/-17, APACHE II score was 25+/-12 and MODS ratio was 6+/-4. The mean duration of stay was 40+/-25 days, the mortality rate was $35 \%$. The average calorie intake was $2300+/-600 \mathrm{kcal}$. There was a weight gain and an increase of the Body Mass Index either in surviving and dead patients. There was an initial increase of the C-reactive protein and the orosomucoid rate during the acute phase of aggression followed by a progressive decrease. The nutritional proteins (albumin, prealbumin, retinol binding protein) were always low, despite a progressive increase. The PINI was initially high and decreased progressively but remained high (> 20). The Albumin and Prealbumin rate were correlated with the MODS ratio. There was a correlation between the orosomucoid rate and the organ failure. The PINI was correlated to the MODS ratio and to the IGS II but not with mortality. Conclusions The malnutrition in surgical patients at intensive care unit has an early onset and is always severe. Biological markers and PINI are correlated with organ failure but not with mortality.
\end{abstract}

Keywords: Nutrition Status, Inflammation, Biological Markers, Surgical Intensive Care Unit, Enteral Nutrition, PINI, Prognosis

\section{Background}

Recent nutrition surveys in hospitals continue to suggest that upwards of $40-50 \%$ of patients, particularly those in the intensive care unit (ICU), have a moderate to severe degree of malnutrition and it has been shown that this degree of malnutrition has a significant negative impact on clinical outcomes [1].

Malnutrition in critical illness results in loss of body cell mass, alterations in mineral homeostasis, and derangements in organ system function. Typical manifestations of these derangements in critically ill patients include impaired immune function, prolonged dependence on mechanical ventilation, and increased rates of infection. These effects often are difficult to differentiate from the concurrent 
illness and injury, but attempts must be made by practitioners to identify and correct nutritional disorders because they render patients vulnerable to infectious complications, increased health care costs, and lead to increased patient morbidity and mortality [2, 3].

Anthropometric data (skinfold thickness and arm muscle circumference), as well as clinical parameters, while useful in ambulatory patients, are significantly less accurate measures of malnutrition in the critically ill patient, particularly in those who have fluid overload or renal dysfunction [1]. So, other Indices have been proposed to provide a reliable nutritional assessment of critically ill patients in intensive care units.

The prognostic inflammatory and nutritional index (PINI) was proposed by Carpentier and Ingembleek in1985, it combines inflammatory proteins (C-reactive protein and orosomucoid) and nutrition proteins (albumin and transthyretin or prealbumin), to give more value for these biomarkers. Despite a significant interest, this index has never been used for the evaluation of patients in surgical intensive care.

The main aims of our study were: first to evaluate the nutritional status of surgical ICU patients by biological markers, and second, to look for a correlation between biological markers and prognosis using the PINI.

\section{Methods}

Over three months $\left(1^{\text {st }}\right.$ July $2013-30^{\text {th }}$ September 2013) a prospective, observational study was performed in an 18bed medical surgical intensive care unit at Tunis military hospital. The approval of the ethics committee was not necessary given the strictly observational nature of the study. Inclusion criteria were: Patients admitted to surgical ICU and aged over 18 years, nutritional assessment done within 48 hours of admission, Length of stay in ICU more than one week. Were not included in this study: patients with chronic inflammatory disease or renal failure and requiring hemodialysis, patients aged less than 18 years. Patients who died in the first week were excluded from the study

Data collected from the medical charts of the patients and from the treating physicians included gender, age, Body Mass Index at admission $\left(\mathrm{kg} / \mathrm{m}^{2}\right)$, Acute Physiology and Chronic Health Evaluation II (APACHE II) score, simplified acute physiology II (SAPS II) score, index of gravity scale II (IGS II), reasons for hospitalization, complications and length of stay in ICU. Nutritional status on admission was assessed as: malnourished if 2 of the 3 below criteria were present: weight loss $<10 \%$ within the last 6 month, BMI < 19, wasting disease; they were considered «normal » with $\mathrm{BMI}=20-30$, and obese if BMI > 30. Patients were weighted weekly during the stay.

Organ failure was quantified by the Multiple Organ Dysfunction Score (MODS) on admission, and on weekly basis.

Laboratory investigation included the following parameters; C-reactive protein (CRP), orosomucoid, albumin, prealbumin, retinol binding protein (RBP) and transferrin allowing calculation of the prognostic inflammatory and nutritional index (PINI). Such laboratory investigations were evaluated weekly until patients discharge from the ICU.The prognostic inflammatory and nutritional index was calculated using the following formula: PINI = CRP $\mathrm{x}$ Orosomucoid / Albumin $\mathrm{x}$ Prealbumin.

All patients, were initiated on enteral or parenteral (EN or PN) feeding, as early as possible after admission. The daily calorie and protein prescriptions were calculated from standard recommendations (calories $30 \mathrm{kcal} / \mathrm{kg} / \mathrm{d}$, proteins $1.2 \mathrm{~g} / \mathrm{kg} / \mathrm{d}$ ) after making appropriate adjustments for the severity of critical illness and comorbid conditions [4,5]. A meticulous record of the prescription and delivery of the volume, calories, and protein content of enteral (and/or parenteral) nutritional supplements was maintained.

The routes of enteral tube feeding were noted. Methods for checking the position of the tube include air insufflation and auscultation of the epigastrium, and Chest X-ray.

Enteral nutrition was given on continuous pump feeding, which means feeding over a 20 hour period.

To reduce risk of aspiration, the head of the bed was elevated around 30 degrees.

Feeding tubes were flushed with $30 \mathrm{ml}$ of water before and after administering each drug.

Patients were monitored for tolerance of EN, determined by patient complaints of pain and/ or distention, physical exam, passage of flatus and stool and Gastric residual volumes. A residual volume of $300 \mathrm{ml}$ was served as a trigger for withholding $\mathrm{EN}$ if associated with other abnormalities (nausea and vomiting, distention...).

Statistical analysis was performed using SPSS 20.0 statistical software. Continuous variables are expressed as mean \pm standard deviation, while categorical variables are expressed with absolute and relative frequencies. The normality assumption of continuous variables was evaluated using the Kolmogorov Smirnov criterion. The correlation between variables was calculated, the Spearman rank-order correlation was used. Risk factors for mortality were tested first by univariate analysis. Those with a significance level of $\mathrm{p} \leq 0.1$ were included in a logistic regression with Wald method analysis as independent variables. All reported $\mathrm{P}$ values are two-tailed. Statistical significance was set at $\mathrm{P}<$ 0.05 .

\section{Results}

During the study period, 85 patients were admitted to the ICU, of whom 20, who fulfilled all the inclusion criteria, composed the study population. The demographic profile and baseline characteristics of the study population are depicted in Table 1.

Nineteen $(95 \%)$ patients received enteral nutrition, either alone $(\mathrm{n}=15)$ or in combination with parenteral nutrition ( $\mathrm{n}$ =4). Only one patient have received parenteral nutrition 
alone because of the inability to use the gastrointestinal tract.Parenteral nutrition was associated with enteral nutrition to reach the caloric intake target. The average calorie intake was $2300 \pm 600 \mathrm{kcal} / \mathrm{d}$.

On admission 1 patient had a BMI <20, 18 were normal, and 1 had a BMI >27. Repeated weekly weight determinations were available in all patients. During the two first weeks, 18 patients gained weight, 2 were stable (BMI varied similarly).

Complications associated with artificial feeding were: sinusitis and related upper respiratory infections, which are reported in $20 \%$ of patients fed through nasoenteric tubes, aspiration of gastric contents and the subsequent development of pneumonia, reported in $15 \%$ of patients and diarrhea, occurred in $25 \%$ of patients.

Figures 1 shows kinetics of Biochemical Assessment of patients during ICU stay. In summary, there is an initial increase in the rate of CRP and orosomucoid, followed by a progressive decrease. Among the biochemical variables indicating the state of visceral proteins, there is a gradual increase in the rate of prealbumin, albumin, RBP and transferrin.

Table 1. Demographic Characteristics of Patients.

\begin{tabular}{ll}
\hline Age (mean \pm SD, years) & $\mathbf{5 6} \pm \mathbf{1 1}$ \\
\hline Sex ratio (M / F) & 1.22 \\
Body weight at admission (mean \pm SD, kg) & $82 \pm 12$ \\
Body Mass Index at admission (mean \pm SD ,kg / & $29 \pm 5$ \\
$\mathrm{~m}^{2}$ ) & $48 \pm 17$ \\
SAPS II at admission( mean \pm SD) & $25 \pm 12$ \\
APACHE II at admission(mean \pm SD) & $6 \pm 4$ \\
MODS on admission(mean \pm SD) & $48 \pm 17$ \\
IGS II at admission( mean \pm SD) & \\
Main reason for ICU admission, (n, \%) & $13(65 \%)$ \\
Trauma & $6(30 \%)$ \\
surgical pathologies & $1(5 \%)$ \\
severe burns & $20(100 \%)$ \\
Mechanical ventilation (n, \%) & $14(70 \%)$ \\
Vasoactive drug therapy (n, \%) & \\
Routes of enteral tube feeding & $18(90 \%)$ \\
Nasogastric tube feeding & $5(25 \%)$ \\
Gastrostomy & $1(5 \%)$ \\
jejunostomy & $40 \pm 25$ \\
Length of stay in ICU (mean \pm SD, days)) & $2300 \pm 600$ \\
Caloric intake (mean \pm SD ,kcal / d) & $7(35 \%)$ \\
Mortality (n, \%) & \\
\hline
\end{tabular}

SAPS II: Simplified Severity Index II, APACHE II, Acute Physiology And Chronic Health Evaluation II, MODS: Multiple Organ Dysfunction Score, IGS II: index of gravity scaleII, ICU: intensive care unit.
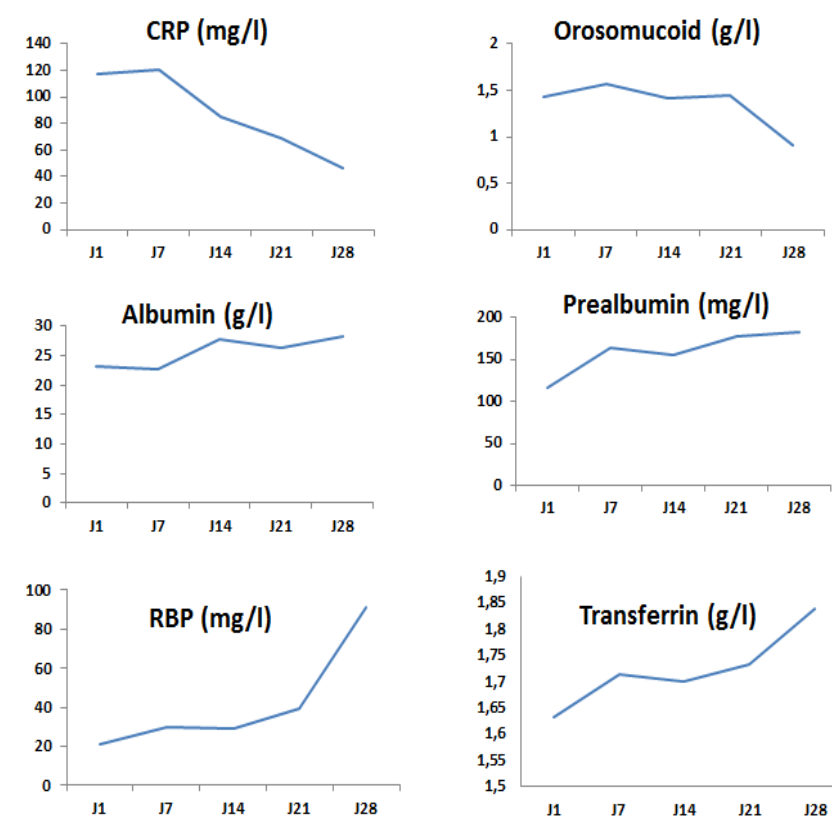

Figure 1. Kinetics of biochemical variables.

The PINI was initially high and decreased progressively but remained high (> 20) (Figure 2).

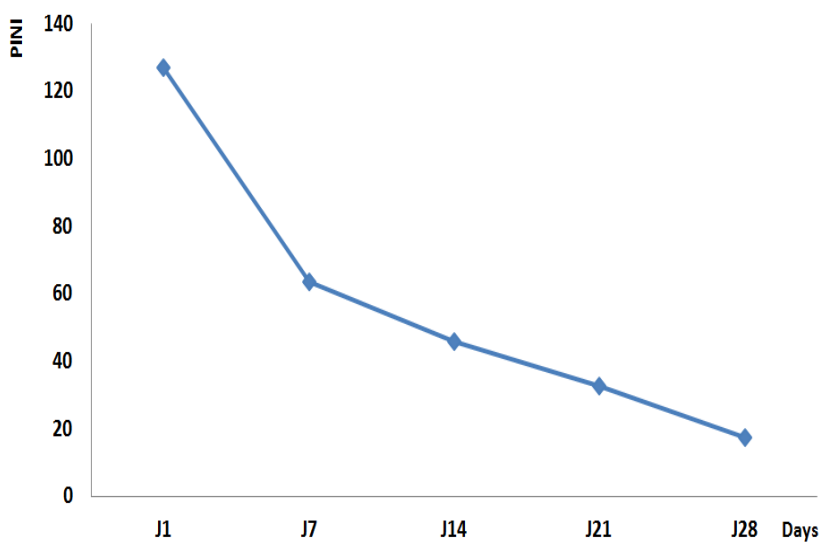

Figure 2. Evolution of PINI during hospitalization.

A positive correlation was found between IGS II, MODS and PINI (Figure 3) 


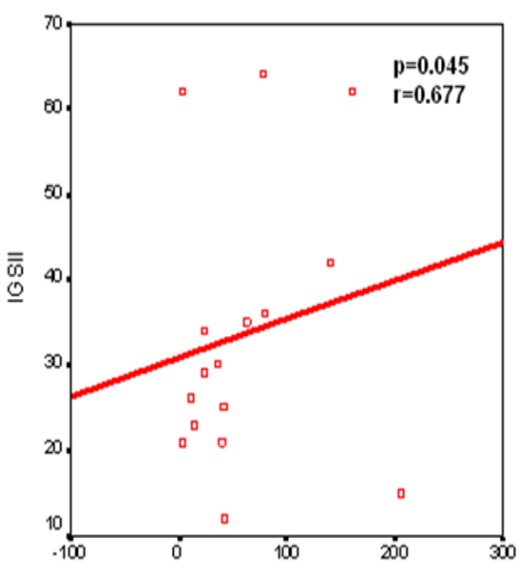

PINI

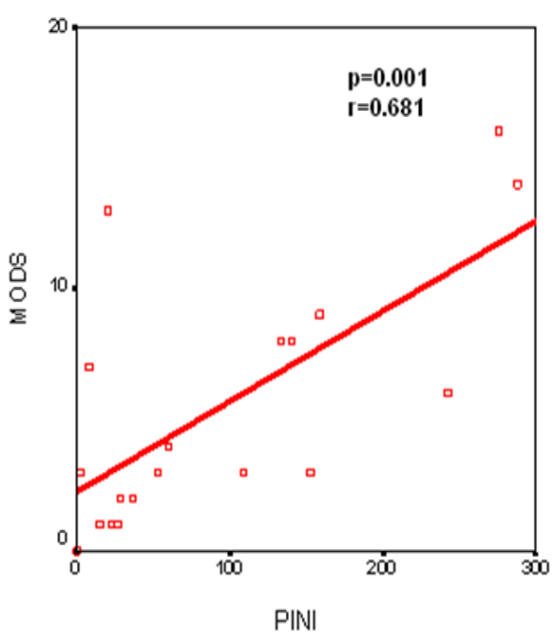

Figure 3. Correlation between IGS II, MODS and PINI.

A negative correlation was found between MODS and nutritional proteins (albumin and Prealbumin) (figure 4).
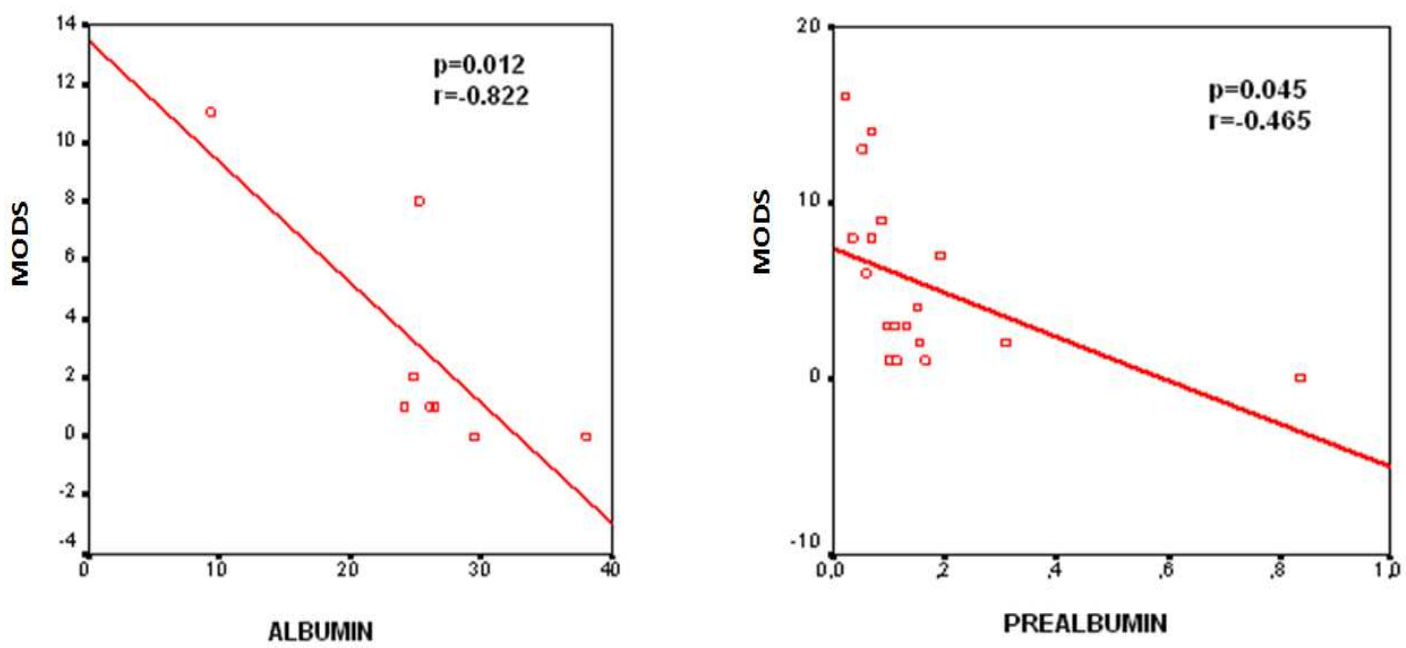

Figure 4. Correlation between albumin, prealbumin and MODS.

Aiming to examine which parameters could predict mortality, we performed an univariate and a multivariate analysis including all collected data considered as potential risk factors. Only age was found to be significant (Table 2).

Table 2. Risk factors of mortality: Univariate and multivariate analysis.

\begin{tabular}{llll}
\hline Risk factors & Died $(\mathbf{n}=\mathbf{7})$ & Survived $(\mathbf{n}=\mathbf{1 3})$ & $\begin{array}{c}\text { P,univariate analysis } \\
\text { analysis }\end{array}$ \\
\hline Age (mean $\pm \mathrm{SD}$, years) & $63 \pm 8$ & $53 \pm 10$ & 0.027 \\
BMI (mean \pm SD ,kg / m $\left.{ }^{2}\right)$ & $30 \pm 5$ & $28 \pm 4$ & 0.617 \\
SAPS II at admission( mean \pm SD) & $50 \pm 17$ & $47 \pm 12$ & 0.774 \\
APACHE II at admission(mean \pm SD) & $28 \pm 12$ & $24 \pm 12$ & 0.534 \\
MODS on admission(mean \pm SD) & $7 \pm 4$ & $6 \pm 3$ & 0.206 \\
IGS II at admission( mean \pm SD) & $48 \pm 17$ & $46 \pm 15$ & 0.645 \\
Length of stay in ICU (mean \pm SD, days) & $46 \pm 25$ & $42 \pm 20$ & 0.530 \\
PINI & 63.42 & 144.42 & 0.101 \\
Albumin (mean,g/l) & 23.25 & 27.81 & 0.974 \\
Prealbumin (mean,mg/l) & 0.12 & 0.16 & 0.625 \\
RBP (mean,mg/l) & 0.022 & 0.030 & 0.288 \\
CRP (mean,mg/l) & 98.13 & 125.48 & 0.317 \\
Orosomucoid (mean,g/l) & 2.12 & 1.72 & 0.741 \\
\hline
\end{tabular}

BMI: Body Mass Index at admission, SAPS II : simplified acute physiology score II, APACHE II : Acute Physiology and Chronic Health Evaluation II, MODS : Multiple Organ Dysfunction Score, IGS II : index of gravity scale II, PINI : prognostic inflammatory and nutritional index, RBP : retinol binding protein, CRP : C-reactive protein. 


\section{Discussion}

This study examined the nutrition support of 20 very ill ICU patients with extensive ICU and hospital stays. In practice nineteen $(95 \%)$ patients received enteral nutrition, either alone $(n=15)$ or in combination with parenteral nutrition $(\mathrm{n}=4)$. Only one patient have received parenteral nutrition alone. Hill et al. reported in 1995 of nutritional support being given to $43 \%$ of ICU patients [6] In their survey $46 \%$ received only parenteral and $34 \%$ only enteral nutrition; $4 \%$ were fed orally and $16 \%$ were fed in more than one way. In 1997, Berger et al. reported that $40 \%$ of surgical ICU patients received nutritional support: $10 \%$ were on parenteral and $30 \%$ on enteral nutrition [7]. The European ICU survey by Preiser et al. revealed that twothirds of patients were receiving nutritional support: $58 \%$ enteral, $23 \%$ parenteral, and $19 \%$ a combination of the two [8]. Albert et al. reported en 2002 that nutritional support was given to $69 \%$ of ICU patients; enteral nutrition alone was given to $58 \%$ patients. Parenteral nutrition alone was given to $17 \%$ and combination of parenteral and enteral nutrition to $5 \%$ of the ICU patients [9].

The physical exam and anthropometry supply important information when evaluating the nutritional state of a patient. Interview and physical examination offer a contact with the patient that cannot be reproduced in the numbers of laboratory tests. The body mass index (BMI) is another simple indicator of the nutritional state. Although values between 18.5 and $24.9 \mathrm{~kg} / \mathrm{m}^{2}, 19$ considered eutrophic, less than $20 \mathrm{~kg} / \mathrm{m}^{2}$ are indicative of malnutrition and associated to a significant increase of mortality in different types of patients [10]. An unintentional loss of body mass greater than $10 \%$ in the last six months or a more rapid loss are prognostic of clinical evolution 21 and classical signs of malnutrition.2 However, it may be difficult to determine the real loss of body mass in sick individuals, because of poor accuracy[11].

Nutritional status can also be assessed by measuring the visceral (or constitutive) protein pool, the acute-phase protein pool, nitrogen balance, and resting energy expenditure (REE). Albumin, which has a large pool and much longer half-life (14-20 days), is not indicative of the immediate nutritional status and may be skewed by changes in fluid status. Serum albumin concentration may be affected by albumin infusion, dehydration, sepsis, trauma, and liver disease, and it is independent of nutritional status. Thus, its reliability as a marker of visceral protein status is questionable. Prealbumin, (also known as transthyretin or thyroxine-binding prealbumin) is a stable circulating glycoprotein synthesized in the liver. It binds with retinol-binding protein and is involved in the transport of thyroxine and retinol. Prealbumin, so named by its proximity to albumin on an electrophoretic strip, has a half-life of 24 to 48 hours and reflects more acute nutritional changes. Prealbumin concentration is diminished in liver disease. Prealbumin is readily measured in most hospitals and is a good marker for the visceral protein pool [12].

For appropriate interpretation, biochemical assessment of protein status should take in consideration the metabolic response to stress and its effect(s) on serum proteins. In this regard, certain serum proteins such as albumin, transferrin, pre-albumin and retinol-binding protein (RBP) are the socalled negative acute phase proteins, wheras C-reactive protein (CRP), ceruloplasmin and various others are positive acute phase proteins. It should also be borne in mind that nitrogen balance assessment is the only biochemical parameter that truly reflects visceral and somatic protein pools. When utilizing plasma protein for the assessment of protein status, the non-nutritional factors that are known to affect plasma proteins concentration need to be considered. Such factors include biological variation, physiological function, hydration status, patient posture at phlebotomy, hepatic and renal function and the acute phase response [13, 14, 15, 16].

Albumin, although a poor indicator of nutritional status in the critically ill patient is a sensitive indicator of morbidity, mortality and length of hospitalization [17] and, in the short term, can be used as a marker of injury and metabolic stress during the acute phase response. In trauma patients an albumin of $\leq 26 \mathrm{~g} / \mathrm{L}$ has been shown to be a significant independent predictor of mortality and morbidity. The combination of a low albumin level and increased age was the most predictive of infection and mortality [18]. Pre-albumin correlates with short term changes in protein energy malnutrition and is a marker of protein intake. Two recent studies though indicate that prealbumin does not respond sensitively to nutrition support [14] particularly during the early period of the acute phase response due to the delayed return to anabolic status [14]. It is, however, a good marker of the systemic inflammatory response [14]. Only in the presence of stable inflammatory parameters does pre-albumin reflect adequacy of nutrition support [15]. The same difficulties in interpretation emerged in a study on retinol-binding protein [16.]In our study the Albumin and Prealbumin rate were correlated with organ failure but not with mortality.

CRP is a sensitive but not a specific marker of inflammation. An abnormally high plasma CRP concentration is found in association with a wide variety of disorders, including infection, trauma, inflammatory arthritis and neoplasms [19]. Moderate elevations in CRP values may also reflect obesity, cigarette smoking or diabetes mellitus. CRP baseline values were also directly related to total mortality [20]. However, there is no convincing argument to link CRP with nutritional status [21].

The Prognostic Inflammatory Nutritional Index (PINI= [Orosomucoid $\times$ C-reactive protein $(\mathrm{CRP})$ ] divided by [albumin $\times$ prealbumin]) is a simple clinical assessment tool which aggregates two blood markers of inflammation (CRP and AAG) with two of nutrition (albumin and 
prealbumin) into a single score. The prognostic value of the score is as follows: $>30=$ life risk, $21-30=$ high risk, $11-$ $20=$ medium risk, $1-10=$ low risk and $<1=$ minimal risk; normal, healthy individual [22].

PINI has been found to be a reliable indicator of both nutritional status and prognosis in trauma, burns, and infection. However, it has been little evaluated in ICU [23, 24]. The mean PINI score was remarkably elevated and even higher than the mean PINI scores reported in critically ill intensive care patients [22]. The PINI was correlated to the MODS ratio and to the IGS II but not with mortality.

Findings from this investigation should be interpreted with caution. Although our analysis captured severity of illness, route of feeding, BMI, sex, and age, the results may have been confounded by unidentified and unmeasured clinical factors and individual patient characteristics that impacted percent goal of kilocalories delivered and patient outcomes in our regression model. For example, one limitation to this study is that some patients who received more kilocalories may have been hyperglycemic (data not captured), which may have negatively impacted patient outcomes. In addition, due to our limited overall sample size and the small sample size that were generated by the classification, a prudent approach should be taken when discerning between surgical and medical populations in regard to percent goal and clinical outcomes.

Another limitation was that events that led to discrepancies between calorie prescription and delivery were not specifically recorded in the current study

In addition, there are no clearly defined measures of outcome in critically ill patients that can be correlated to nutrition directly, and this makes the process of conducting clinical trials in the field of nutrition difficult. Association of low caloric intake with nosocomial bloodstream infections in medical ICU patients has been observed previously and may serve as another outcome measure, although in this case also it was unclear whether the relationship was causal or not.

\section{Conclusions}

The malnutrition in surgical patients at ICU has an early onset and is always severe. Albumin, prealbumin and PINI are correlated with organ failure but not with mortality.

Future studies involving a larger number of patients and those that are designed specifically to determine the optimal timing and level of nutritional support to critically ill patients could help answer some of the unanswered questions that exist today in relation to critical care nutrition.

\section{Abbreviations}

ICU: intensive care unit; PINI: prognostic inflammatory and nutritional index; BMI: Body Mass Index at admission; APACHE: Acute Physiology and Chronic Health Evaluation score; SAPS: simplified acute physiology score;
IGS: index of gravity scale; MODS: Multiple Organ Dysfunction Score; CRP: C-reactive protein; RBP: retinol binding protein; $\mathrm{EN}$ : enteral nutrition; $\mathrm{PN}$ : parenteral nutrition; REE: resting energy expenditure.

\section{Authors' Contributions}

$\mathrm{ZH}, \mathrm{HG}$ and MF devised the study protocol. ZH, IN, ZO and NS collected and analyzed the data. ZH, IL performed statistical analysis. ZH and HG wrote the manuscript. All authors have read and approved the manuscript for publication.

\section{References}

[1] Ait Hssain A, B Souweine, NJ Cano : Pathophysiology of malnutrition in ICU. Nutr Clin Metaboli 2011 ,25:29-35.

[2] P Erny: Nutrition assaulted: consensus conference. Ann Fr Anesth Réanim 1998,17:1274-84.

[3] Herridge MS, Cheung AM, Tansey CM et al: One-year outcomes in Survivors of the acute respiratory distress syndrome. N Engl J Med 2003, 348:683-93.

[4] Kreymann KG, Berger MM, Deutz NE, Hiesmayr M, Jolliet P, Kazandjiev G, Nitenberg G, van den Berghe G, Wernerman J, Ebner C et al: ESPEN Guidelines on Enteral Nutrition: Intensive care. Clin Nutr 2006 , 25(2):210-223.

[5] McClave SA, Martindale RG, Vanek VW, McCarthy M, Roberts P, Taylor B, Ochoa JB, Napolitano L, Cresci G:Guidelines for the Provision and Assessment of Nutrition Support Therapy in the Adult Critically Ill Patient:Society of Critical Care Medicine (SCCM) and American Society for Parenteral and Enteral Nutrition(A.S.P.E.N.). Jpen 2009, 33(3):277-316.

[6] Hill SA, Nielsen MS, Lennard-Jones JE: Nutritional support in intensive care units in England and Wales: a survey. Eur J Clin Nutr 1995,49:371-8.

[7] Berger MM, Chiole'ro RL, Pannatier A, Cayeux MC, Tappy L: A 10-year survey of nutritional support in a surgical ICU: 1986-1995. Nutrition 1997,13:870-7.

[8] Preiser JC, Berre' J, Carpentier Y, Joilliet P, Pichard C,Van Gossum A et al: Management of nutrition in European intensive care units: results of a questionnaire. Intens Care Med 1999,25:95-101.

[9] Albert H. Verhage, Adrie C.M. van Vliet: Clinical practice of nutritional support in Dutch intensive care units: a survey. Eur J Intern Med 2002, 13(8):496-499.

[10] Campillo B, Paillaud E, Uzan I, Merlier I, Abdellaoui M, Perennec $\mathrm{J}$, et al:Value of body mass index in the detection of severe malnutrition: influence of the pathology and changes in anthropometric parameters. Clin Nutr 2004,23(4):551-9.

[11] Jeejeebhoy KN: Nutritional assessment. Nutrition 2000,16(7-8):585-90.

[12] Robinson MK, Trujillo EB, Mogensen KM, et al: Improving nutritional screening of hospitalized patients: the role of prealbumin. J Parenter Enteral Nutr 2003, 27(6):389-95. 
[13] Gariballa S, Forster S:Effects of acute-phase response on nutritional status and clinical outcome of hospitalized patients. Nutrition $2006,22(7-8): 750-7$.

[14] Devakonda A, George L, Raoof S, Esan A, Saleh A, Bernstein LH: Transthyretin as a marker to predict outcome in critically ill patients. Clin Biochem $2008,41(14$ 15):1126-30.

[15] Raguso CA, Dupertuis YM, Pichard C:The role of visceral proteins in the nutritional assessment of intensive care unit patients.Curr Opin Clin Nutr Metab Care 2003,6(2):211-6.

[16] López-Hellin J, Baena-Fustegueras JA, Schwartz-Riera S, García-Arumí E: Usefulness of short-lived proteins as nutritional indicators surgical patients. Clin Nutr $2002,21(2): 119-25$.

[17] Higgins PA, Daly BJ, Lipson AR, Su-Er G:Assessing nutritional status in chronically ill adult patients. Am J Crit Care 2006,15:1-99.

[18] Sung J, Bochicchio GV, Joshi M, et al: Admission serum albumin is predicitve of outcome in critically ill trauma patients. Am Surg $2004,70(12): 1099-102$.
[19] Mahmoud FA, Rivera NI :The role of C-reactive protein as a prognostic indicator in advanced cancer. Curr Oncol Rep $2002,4: 250-255$.

[20] Koenig W, Pepys MB :C-reactive protein risk prediction: low specificity, high sensitivity. Ann Intern Med $2002,136: 550-552$.

[21] Prins A: Nutritional assessment of the critically ill patient. S Afr J Clin Nutr 2010,23(1):11-18.

[22] Ingenbleek Y, Carpentier YA :A prognostic inflammatory and nutritional index scoring critically ill patients. Int $\mathrm{J}$ Vitam Nutr Res $1985,55: 91-101$.

[23] Ho SY, Guo R Hf, HHW Chen, CJ Peng: Nutritional predictors of survival in terminally ill cancer patients.J Formosa Med Assoc 2003,102:544-550.

[24] Nelson K, Walsh D:The cancer anorexia-cachexia syndrome; a survey of the prognostic inflammatory and nutritional index (PINI) in advanced disease. J Pain Symptom Manage 2002,24:424-428. 